\title{
Incidence and prevalence of neurological disorders associated with HIV since the introduction of highly active antiretroviral therapy (HAART)
}

\author{
Matthias Maschke, Oliver Kastrup, Stefan Esser, Birgit Ross, Ulrich Hengge, \\ Andreas Hufnagel
}

\begin{abstract}
Objective-To determine the change of incidence and prevalence of neurological disorders caused by the human immunodeficiency virus (HIV) and opportunistic infections in HIV positive patients under treatment since the introduction of highly active antiretroviral therapy (HAART). Methods-The data of all HIV infected patients were retrospectively analysed, who were examined in the HIV outpatients clinic of the neurological department of the University Clinic Essen between 1995 and $1998(n=563$, total number of visits=735). Data from identified patients were divided into two groups according to the time of examination from 1995 to 1996 (334 visits) and from 1997 to 1998 (401 visits). The incidence and prevalence of neurological disorders were statistically compared between both time intervals.

Results-Significantly more patients received HAART in 1997-8 $(\mathrm{p}<0.001)$ and mean CD4+ cell count was significantly higher in 1997-8 $(\mathrm{p}<0.001)$. The prevalence of HIV associated dementia and HIV associated polyneuropathy were significantly lower in 1997-8 (both: $p=0.02$ ) and the incidence of toxoplasma encephalitis decreased from $5.7 \%$ in $1995-6$ to $2.2 \%$ in 1997-8 $(p=0.015)$. Based on the small number of patients significant changes in HIV associated myopathy, progressive multifocal leukoencephalopathy, cryptoccocal meningitis, and cytomegalovirusencephalitis could not be detected.

Conclusion-The prevalence of the most frequent HIV associated neurological disorders and incidence of toxoplasma encephalitis decreased since the introduction of HAART. This may be due to the improvement of immunostatus by HAART as demonstrated by the higher CD4+ cell count in the later time interval. Direct antiretroviral effects within the nervous system may be considered causative as well. The prevalence and incidence of HIV associated neurological disorders and opportunistic CNS infections decreased after introduction of HAART.
\end{abstract}

Correspondence to: Dr Matthias Maschke

matthias.maschke@uni-essen.de (F Neurol Neurosurg Psychiatry 2000;69:376-380)

Received 13 September 1999

and in final form

21 March 2000

Accepted 12 April 2000
Infection with HIV is often complicated by neurological disorders in the later phases of the disease. In the time before antiretroviral combination therapy in the form of the so called highly active antiretroviral therapy (HAART) was available, neurological complications were the initial manifestation of the acquired immunodeficiency syndrome (AIDS) in $7 \%-20 \%$. It is reported that $39 \%-70 \%$ of all patients with AIDS or symptomatic HIV infection developed neurological disorders. ${ }^{1} \mathrm{Re}-$ cently, two studies reported a decrease in the incidence of opportunistic CNS infections such as toxoplasma encephalitis after the initiation of HAART. ${ }^{23}$ However, whether HAART leads to a reduction in incidence and prevalence of HIV associated dementia and HIV associated polyneuropathy remains unclear. This study investigated the incidence and prevalence of HIV related disorders before and after introduction of HAART between the years 1995 and 1998.

\section{Patients and methods}

All patients with known HIV infection, who were examined between 1995 and 1998 in the neurological department of the University Clinic of Essen, were retrospectively identified and included in the analysis. The total number of identified patients was 563 with a total number of 735 visits during the whole time period. According to the time of examination the data of the study population were divided into the time interval from 1995-6 (334 visits) and from 1997-8 (401 visits); 80.3\% (452) of patients were seen only once in the whole time period, whereas $17.7 \%$ (111) were seen more than once. Incidence data from patients who were seen more than once were counted only in the year of diagnosis. By contrast, prevalence data (for example, HIV associated dementia, distal symmetric polyneuropathy, and myopathy) were counted for each visit, but only once for each year. The referral pattern of the patients did not change across the years. Basic characteristics (sex, age, disease duration, CD4+ cell count, antiretroviral therapy) were obtained from chart records at the neurological department and at the two referring departments of the same institution (department of dermatology, department of internal medicine). Basic characteristics are summarised in table 1. Briefly, mean CD4+ cell counts were significantly higher in 1997-8. Significantly more patients received 
Table 1 Basic characteristics of patient populations in 1995-6 and 1997-8

\begin{tabular}{llll}
\hline & $\begin{array}{l}1995-6 \\
(n=334)\end{array}$ & $\begin{array}{l}1997-8 \\
(n=401)\end{array}$ & $p$ Value \\
\hline Sex: & $81.1 \%$ & $84.5 \%$ & $\mathrm{NS}^{\star}$ \\
$\quad$ Male & $18.9 \%$ & $15.5 \%$ & \\
$\quad$ Female & $10.2 \%$ & $15.9 \%$ & $\mathrm{NS}^{\star}$ \\
Mean age (SD) & $38.6(9.5)$ & $40.7(10.6)$ & $0.004 \dagger$ \\
Mean disease duration/y (SD) & $5.2(3.5)$ & $5.0(3.7)$ & $\mathrm{NS} \dagger$ \\
Mean CD4+ cell count/ $\mu$ l(SD) & $214(212)$ & $335(267)$ & $<0.001 \dagger$ \\
Proportion of patients under HAART & $34.2 \%$ & $79.3 \%$ & $<0.001^{\star}$ \\
\hline
\end{tabular}

HAART $=$ Highly active antiretroviral therapy; iv=intravenous.

${ }^{\star} \chi^{2}$ test; $\dagger t$ test.

HAART in 1997-8. In 1995-6, the antiretroviral therapy consisted mainly of a combination of two nucleoside reverse transcriptase inhibitors (zidovudine (AZT)+lamivudine (3TC) and AZT+zalcitabine (ddC), AZT+didanosine (ddI), ddI+3TC). After the introduction of protease inhibitors, and thus introduction of HAART, in 1996, therapy consisted of a combination of one protease inhibitor with two reverse transcriptase inhibitors (AZT+3TC+saquinavir (SQV) or indinavir, stavudine $(\mathrm{d} 4 \mathrm{~T})+\mathrm{ddI}+\mathrm{SQV}$ or indinavir), a combination which was not different from the HAART used in 1997-8. The use of prophylactic antibiotics against toxoplasma encephalitis did not change from 1995 to 1998 . Every patient with a CD4+ cell count below $250 / \mu 1$ received cotrimoxazole $(400 \mathrm{mg}$ sulfamethoxazole $+80 \mathrm{mg}$ trimethoprim once a day). In the case of intolerance, patients received pyrimethamine (25 $\mathrm{mg}$ twice daily+15 mg folinic acid twice a week) in combination with pentamidine inhalations (200 mg every 4 th week).

HIV associated dementia was diagnosed according to the guidelines of the AIDS Task Force of the American Academy of Neurology ${ }^{4}$ and in the absence of other causes of dementia (toxic and metabolic encephalopathy, progressive multifocal leukoencephalopathy, other opportunistic CNS infections). The diagnosis of HIV associated distal symmetric sensory polyneuropathy was established in the case of distal symmetric numbness or paraesthesias, reduced or absent ankle reflexes, loss or marked impairment of vibration sense, and typical pathological sensory and/or motor nerve conduction studies. Drug induced polyneuropathy was considered in patients receiving didanosine (ddI), zalcitabine (ddC), and stavudine (d4T) and improvement of symptoms after reduction or termination of these drugs. The diagnosis of HIV associated myopathy was made if proximal myalgia, increased creatine kinase, or myopathic changes on EMG were present and the patients were not on azidothymidine (AZT) at the time

Table 2 Basic characteristics of patients with HIV associated dementia

\begin{tabular}{|c|c|c|c|}
\hline & $1995-6$ & $1997-8$ & $p$ Value \\
\hline Number of patients & 57 & 45 & \\
\hline Prevalence & $17.1 \%$ & $11.2 \%$ & 0.022 \\
\hline Mean CD $4+$ cell count $/ \mu \mathrm{l}(\mathrm{SD})$ & $178(245)$ & $271(279)$ & \\
\hline Mean disease duration/y (SD) & $6.9(3.1)$ & $6.2(3.7)$ & \\
\hline Proportion of patients under HAART & $25.5 \%$ & $95.0 \%$ & \\
\hline iv Drug misuse & $33.3 \%$ & $26.7 \%$ & \\
\hline
\end{tabular}

HAART=Highly active antiretroviral therapy; iv=intravenous. of diagnosis. Toxoplasma encephalitis was suspected in patients with focal signs and symptoms, multifocal enhancing mass lesions in MRI or CT, positive toxoplasma antibodies, and improvement of symptoms after initiation of unidirectional antitoxoplasmosis therapy (therapy with pyrimethamine and sulfadiazine or clindamycine). Cryptococcal meningitis was considered in the presence of typical symptoms and detection of cryptococcal antigen or direct identification of cryptoccal organisms in the CSF. Progressive multifocal leukoencephalopathy (PML) was diagnosed in patients with progressive focal signs and symptoms, decline in cognitive function, multifocal nonenhancing white matter lesions on MRI, positive JC virus polymerase chain reaction in CSF, or positive necropsy findings. Cytomegalovirus (CMV) encephalitis was assumed in the presence of focal signs and symptoms, positive CMV-DNA in plasma and CSF, and nonspecific pathological MR findings such as ventriculomegaly, periventricular enhancement, or cortical and subcortical inflammation and positive necropsy. Primary cerebral lymphoma was diagnosed in patients with unifocal enhancing mass lesions shown by MRI and positive findings on brain biopsy.

In a statistical analysis, the 2 year incidences of opportunistic infections were compared between both time intervals (1995-6 $v$ 19978). For HIV associated dementia, myopathy, and polyneuropathy the 2 year prevalence was calculated and compared between both time intervals by $\chi^{2}$ tests. Numerical parameters were compared by means of the $t$ test. p Values $<0.05$ were considered significant.

\section{Results}

HIV ASSOCIATED DEMENTIA

The prevalence of HIV associated dementia decreased markedly from 1995-6 to 1997-9 (table 2). In 1995-96 HIV associated dementia was diagnosed in 57 patients $(17.1 \%$ of all patients in this interval), whereas in 1997-8 the diagnosis was established in 45 patients $(11.2 \%)$. This difference was significant $(p=0.022)$.

Patients with the diagnosis of HIV associated dementia had lower CD4+ cell counts than patients without disease, although this trend was non-significant (table 2). In 1997-8 significantly more patients with HIV associated dementia received HAART in comparison with the remaining subjects $(95 \% v 77 \%, \mathrm{p}=0.009)$. By contrast, the proportion of patients receiving HAART was not significantly different between patients with HIV associated dementia and others in $1995-6(25.5 \%$ v $35.9 \%$, $\mathrm{p}=0.151)$. The disease duration of patients with HIV associated dementia was significantly longer compared with patients without dementia both in 1995-6 and 1997-8 ( $p<0.001$ and $\mathrm{p}=0.018$ ). The proportion of patients with HIV associated dementia, who reported intravenous drug misuse, was higher in comparison with patients without dementia (1995-6: 33.3\% v $12.5 \%$; 1997-8: $26.7 \%$ v $8.1 \%)$. 
HIV ASSOCIATED DISTAL SYMMETRIC SENSORY POLYNEUROPATHY

The prevalence of HIV associated distal symmetric sensory polyneuropathy (DSP) showed a decline from 1995-6 to 1997-8. In 1995-6 DSP was found in 142 patients $(42.5 \%)$ compared with the lower frequency of 138 patients $(34.4 \%)$ in $1997-8$ (table 3 ). The difference between both intervals was significant $(p=0.022)$.

Patients with DSP had a significantly lower CD $4+$ cell count $(p<0.001)$ and a significantly longer disease duration $(p=0.005)$ in 1997-8 compared with patients without DSP, whereas there were no significant differences between patients with and without DSP in 1995-6 $(p=0.2$ for $C D 4+$ cell count and $p=0.09$ for disease duration, table 3 ). Significantly more patients with DSP received HAART compared with patients without DSP in $1997-8(90.3 \%$ v $73.2 \%, \mathrm{p}>0.001)$. By contrast, there was no significant difference in the proportion of patients with DSP and HAART and patients without DSP and HAART in 1995-6 (38.6\% v $30.9 \%, \mathrm{p}=0.15$ ).

Suspected drug induced polyneuropathy as the cause of distal symmetric polyneuropathy was found in 43 patients in 1997-8 (31.2\% of all patients with DSP). In 1995-6 drug induced DSP was diagnosed in 29 patients $(20.4 \%$ of all patients with DSP). The proportion of patients with drug induced DSP was significantly higher in 1997-8 compared with the other time interval $(\mathrm{p}=0.04)$.

HIV ASSOCIATED MYOPATHY

The overall prevalence of HIV associated myopathy was low in our study population. In 1995-6 only eight patients $(2.4 \%)$ showed signs and symptoms of HIV associated myopa-

Table 3 Basic characteristics of patients with HIV associated distal symmetric sensory polyneuropathy

\begin{tabular}{|c|c|c|c|}
\hline & 1995-6 & $1997-8$ & $p$ Value \\
\hline Number of patients & 142 & 138 & \\
\hline Prevalence & $42.5 \%$ & $34.4 \%$ & 0.022 \\
\hline 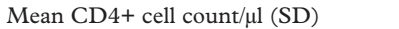 & $194(198)$ & $260(230)$ & \\
\hline Mean disease duration/y (SD) & $5.6(3.6)$ & $5.7(3.6)$ & \\
\hline Proportion of patients under HAART & $38.6 \%$ & 90.3 & \\
\hline Proportion of drug induced DSP & $20.4 \%$ & $31.2 \%$ & \\
\hline
\end{tabular}

HAART $=$ Highly active antiretroviral therapy; DSP=distal symmetric sensory polyneuropathy.

Table 4 Basic characteristics of patients with toxoplasma encephalitis

\begin{tabular}{llll}
\hline & $1995-6$ & $1997-8$ & $p$ Value \\
\hline Number of patients & 19 & 9 & \\
Incidence & $5.7 \%$ & $2.2 \%$ & 0.015 \\
Mean CD4+cell count/ $\mu$ l (SD) & $87(94)$ & $117(90)$ & \\
Mean disease duration/y (SD) & $4.8(3.0)$ & $5.2(3.7)$ & \\
Proportion of patients under HAART & $25 \%$ & $67 \%$ & \\
\hline
\end{tabular}

HAART=highly active antiretroviral therapy.

Table 5 Frequencies of other opportunistic CNS infections than toxoplasma encephalitis and frequency of primary CNS lymphoma

\begin{tabular}{lll}
\hline & $1995-6(n(\%))$ & $1997-8(n(\%))$ \\
\hline Cryptococal meningitis & $3(0.9)$ & $1(0.2)$ \\
Progressive multifocal leukoencephalopathy & $2(0.6)$ & $2(0.5)$ \\
CMV encephalitis & $4(1.2)$ & $6(1.5)$ \\
Tuberculosis meningitis & $3(0.9)$ & $3(0.7)$ \\
Primary CNS lymphoma & $2(0.5)$ & $1(0.2)$ \\
\hline
\end{tabular}

$\mathrm{CMV}=$ cytomegalovirus. thy, whereas HIV associated myopathy was diagnosed in six patients (1.5\%) in 1997-8. This small difference was not significant. Basic characteristics of these patients are not presented due to the low prevalence and lack of change between both intervals.

TOXOPLASMA ENCEPHALITIS

Toxoplasma encephalitis was found in 19 patients $(5.7 \%)$ in $1995-6$, whereas only nine patients developed toxoplasma encephalitis in 1997-8 (2.2\%) reflecting a significant decrease in the incidence of toxoplasma encephalitis from $1995-6$ to $1997-8(\mathrm{p}=0.015$, table 4$)$.

The disease duration was not significantly different in patients with toxoplasma encephalitis compared with the remaining patients (both intervals $\mathrm{p}>0.2$ ), but CD4+ cell counts were significantly lower in both 1995-6 $(p=0.01)$ and $1997-8(p=0.03)$. There was no difference in the proportion of patients with toxoplasma encephalitis who received HAART compared with other patients (both intervals $\mathrm{p}>0.2$ ).

\section{PROGRESSIVE MULTIFOCAL}

LEUKOENCEPHALOPATHY, CYTOMEGALOVIRUS ENCEPHALITIS, CRYPTOCOCCAL MENINGITS, AND PRIMARY CNS LYMPHOMA

The incidence of opportunistic CNS infections other than toxoplasma encephalitis was very low in both intervals and there was no significant change between 1995-6 and 1997-8. Primary CNS lymphoma was also found only in a small proportion of patients. Exact frequencies are summarised in table 5.

\section{Discussion}

Opportunistic CNS infection and HIV associated neurological disorders such as HIV associated dementia and HIV associated distal symmetric polyneuropathy often complicate later stages of HIV disease and occur in up to $40 \%-60 \%$ of all patients with AIDS during their lifetime. ${ }^{5}$ Recently, Brodt et $a l^{2}$ and Michaels et $a l^{\beta}$ reported a decline in incidence and prevalence of opportunistic CNS infections, which may be related to the introduction and widespread use of the highly active antiretroviral therapy (HAART) in western countries since 1996. Whether HAART also has an impact on frequency of HIV associated disorders such as HIV associated dementia or HIV associated polyneuropathy remains unclear. The present study focuses on the changes in incidence of opportunistic CNS infections and prevalence of HIV associated disorders (dementia, polyneuropathy, myopathy) since the introduction of HAART.

In our study population, the prevalence of HIV associated dementia (HAD) showed a significant decrease from 1995-6 to 1997-8. This finding is underlined by the results of the Multicentre AIDS Cohort Study (MACS), which recently also reported a trend towards a decreased rate of HAD comparing incidence rates in 1990-2 with $1993-5$ and $1995-7 .{ }^{6}$ In that cohort, incidence rates were $17.4 / 1000$ person-years in 1993-5 14.7/1000 personyears in 1995-7 respectively. However, 
prevalence rates were not reported. In the present study, the higher proportion of patients in 1997-8 compared with 1995-6, who received HAART, may indicate that the decline of HAD may be due to therapy. However, this has to be proved in prospective studies. By contrast, an Australian study found a relative increase of HIV associated dementia as the initial AIDS defining illness after the introduction of HAART (4.4\% from 1992-6, 6.0\% in 1996 , and $6.5 \%$ in 1997). ${ }^{7}$ It has to be taken into account that data about the initial AIDS defining illness do not have to correspond closely with the prevalence data. Moreover, there may be a difference in epidemiological data between western countries due to the different proportion of patients with intravenous drug misuse, which may act as a predisposing factor for the development of HIV associated dementia. In the present study, there was a significantly higher proportion of patients with disease who were on HAART in comparison with patients without disease in 1997-8. This should not be interpreted as an indication that HAART is associated with HAD. There may be two explanations for this fact. Firstly, patients with HAD or DSP have more advanced stages of HIV infection, and thus are more likely to be on HAART. Secondly, patients with HAD or DSP are more prone to have disease than patients without any symptoms and thus are more likely to consider taking drugs and consult an HIV specialist regularly.

Whether HAART leads to a direct inhibition of HIV within the CNS or acts via improvement of the general immunostatus is a matter of debate. HAART mostly includes at least one protease inhibitor, substances that have only poor CSF penetration. Despite this shortcoming, inhibitors of the reverse transcriptasenamely, azidothymidine (AZT), dideoxyinosine (ddI), and stavudine (d4T)-show sufficient $\mathrm{CSF} /$ plasma ratios and a preliminary study has demonstrated that suppression of HIV RNA within the CSF can occur with indinavir ${ }^{8}$ or with a combination therapy. ${ }^{9}$ In the present study, higher CD4+ cell counts in 1997-8 compared with 1995-6 reflect an improvement in the general immunostatus of patients. Thus, the decrease in the prevalence of HAD may be explained by direct inhibition of HIV within the brain as well as by improvement of immunological indices by HAART. Moreover, HAART may lead to suppression of inflammatory neurotoxins within the brain. This assumption is supported by a study which investigated changes in inflammatory neurotoxins due to HAART in patients with HAD. ${ }^{9}$ That study showed a significant decrease in levels of tumour necrosis factor- $\alpha$, quinolinic acid, and nitric oxide in plasma and CSF. Whether HAART is only preventive or may also reverse cognitive symptoms was examined by a recent study, which noted an improvement of psychomotor speed performance in HIV infected patients who received HAART. ${ }^{10}$ Another remarkable result was the higher mean CD4+ cell count of patients with HAD in the time interval from 1997-98 with a mean CD4 cell count of $271 / \mu 1$ in our study. This was con- firmed by a recently published study by Sacktor et $a l^{6}{ }^{6}$ They reported that the CD4+ cell count nearest to the time of diagnosis of HAD was $>200$ in only $29 \%$ of patients with HAD from $1990-2$, but was $>200$ in $63 \%$ of the HAD cases from 1996-7. This indicates that HAD nowadays may also occur in patients with relatively normal CD4+ cell counts. Furthermore, in the present study there was a higher proportion of patients with intravenous drug misuse in the group of patients with HAD compared with the general population. This finding was also made by others, ${ }^{11}$ but among AIDS cases reported to the CDC, dementia has been found with similar frequency among all risk groups in the United States. ${ }^{12}$ Thus, it remains unclear whether intravenous drug misuse is a risk factor for the development of HAD. However, there is increasing evidence that drugs such as cocaine enhance HIV invasion of the brain by an increase in permeability of the blood-brain barrier. ${ }^{13}$

Another important result of the present study was the significant decline in the prevalence of HIV associated distal symmetric polyneuropathy (DSP) in the time interval from 1997-8. The prevalence rate (34.4\%) during that time interval was lower than prevalence rates reported by studies in the time before the introduction of HAART. ${ }^{14}$ The higher proportion of patients who received HAART in the time interval from 1997-8 compared to the time interval from 1995-6 suggested that the decline in prevalence may be related to HAART. HAART leads to an improvement in CD4+ cell counts, reflected by a higher mean CD4+ cell count in patients of the time interval from 1997-8. Interestingly, a recent study showed a predictive value of CD4+ cell counts for the development of DSP. ${ }^{15}$ In that study, patients with CD4+ cell counts below $750 / \mathrm{mm}^{3}$ had a 1.4 fold greater risk of DSP than those with CD4+ cell counts above $750 / \mathrm{mm}^{3}$. Whether HAART also improves symptoms in patients with DSP has yet to be determined. However, case reports suggested that HAART may be effective in treatment of DSP, which hypothetically may be due to suppression of macrophage activation and neurotoxin production in the peripheral nerve. ${ }^{16}$ Another interesting point in the present study was the increase in the prevalence of toxic neuropathies in the time interval from 1997-8. This might be associated with a frequent use of possible neurotoxic antiretroviral drugs such as stavudine (d4T) and didanosine (ddI), which are not uncommonly used in combination with a protease inhibitor. However, these changes in prevalence should be regarded cautiously in the light of the retrospective character of the study.

The third main point of the present study is the significant decrease in the incidence of toxoplasma encephalitis in the time interval from 1997-8. This result is in keeping with the decline of toxoplasma encephalitis in the past years reported by Brodt et $a l^{2}$, Michaels et $a l^{3}$ and Sacktor et al. ${ }^{6}$ By contrast with patients with HAD and DSP, patients with toxoplasma encephalitis showed a low CD4+ cell count 
both in the time interval from 1995-6 and from 1997-8. This finding indicates that toxoplasma encephalitis commonly occurs in patients with advanced immunosuppression, a fact which has already been found by others in the time period before the introduction of HAART. ${ }^{17-19}$ This decrease in the incidence may be related to HAART, which leads to a smaller proportion of patients with advanced immunosuppression indicated by the higher mean CD4+ cell count in our patient population in the time interval from 1997-8.

The lack of change in cryptococcal meningitis, cytomegalovirus encephalitis, progressive multifocal leukoencephalopathy (PML), and primary CNS lymphoma as well as HIV associated myopathy is probably explained by the low number of patients with these conditions in both the time interval from 1995-6 and from 1997-8. However, others reported a decline in all cytomegalovirus infections after introduction of HAART. ${ }^{2}{ }^{3}$ Whether HAART has an impact on development of PML remains to be investigated, but two recent studies showed an improvement in prognosis of PML due to HAART. ${ }^{20}{ }^{21}$ In the Multicenter AIDS Cohort Study, there was a trend toward a decreased rate of cryptococcal meningitis as well as primary CNS lymphoma after introduction of HAART. ${ }^{6}$

In conclusion, the present study showed a significant decrease in the prevalence of the two most common HIV associated neurological disorders, HIV associated dementia and HIV associated distal symmetric sensory polyneuropathy, after the introduction of highly active antiretroviral therapy (HAART). Moreover, HAD and DSP seems to occur nowadays at a higher range of $\mathrm{CD} 4+$ cell counts. The incidence of toxoplasma encephalitis also showed a decline after introduction of HAART, but still tended to occur regularly in patients with advanced immunosuppression. Further prospective studies are necessary to confirm these results and to identify which combination of antiretroviral agents inhibits best the development of HIV related neurological disorders.

1 Janssen RS. Epidemiology and neuroepidemiology of human immunodeficiency virus infection. In: Berger JR, Levy RM, eds. AIDS and the nervous system, 2nd ed. Philadelphia: Lippincott-Raven 1997:13-37.
2 Brodt HR, Kamps BS, Gute P, et al. Changing incidence of AIDS-defining illnesses in the era of antiretroviral combiAIDS-defining illnesses in the era of an

3 Michaels J, and the Adult Spectrum of Disease Cohort. Opportunistic incidence rates from the Tulane/adult spectrum of disease cohort, 1994-8. Fifth conference on retroviruses and opportunistic infections. Chicago, 1998;1.25.2 .

4 Janssen RS, Cornblath DR, Epstein LG, et al. Human immunodeficiency virus (HIV) infection and the nervous system: report from the American Academy of Neurology
AIDS Task Force. Neurology 1989;39:119-22.

5 Bendok B, Berger JR, Levy RM. Acquired immunodeficiency syndrome and the nervous system: fifteen years
of progress. In: Berger JR, Levy RM, eds. AIDS and the of progress. In: Berger JR, Levy RM, eds. AIDS and the nervous system.

6 Sacktor N, Lyles RH, McFarlane G, et al, and the multicenter AIDS cohort study. HIV-1 related neurological disease incidence change in the era of highly active antiretroviral therapy. Neurology 1999;52:A252.

7 Dore GJ, Correll PK, Li Y, et al. Changes to AIDS dementia complex in the era of highly active antiretroviral therapy. AIDS 1999;12:1249-53.

8 Collier AC, Marra C, Coombs RW, et al. Cerebrospinal fluid indinavir and HIV RNA levels in patients on chronic indinavir therapy. In: Program and abstracts of the Infectious Disease Society of America 35th anmual meeting. San Francisco, CA: 1997; September 13-16:75.

9 Gendelman HE, Zheng J, Coulter CL, et al. Suppression of inflammatory neurotoxins by highly active antiretroviral inflammatory neurotoxins by highly active antiretroviral dementia. F Infect Dis 1998;178:1000-7.

10 Sacktor N, Lyles RH, Skolasky RL, et al, for the multicenter AIDS cohort study (MACS). Combination antiretroviral therapy improves psychomotor speed performance in HIVseropositive homosexual men. Neurology 1999;52:1640-9.

11 Schwartlander B, Horsburgh CR Jr, Hamouda O, et al. Changes in the spectrum of AIDS-defining conditions and decrease in CD4+ lymphocyte counts at AIDS manifestation in Germany from 1986-91. AIDS 1992;6:413-20.

12 Janssen RS, Nwanyanwu OC, Selik RM, et al. Epidemiology of human immunodeficiency virus encephalopathy in the United States. Neurology 1992;42:1472-6.

13 Zhang L, Looney D, Taub D, et al. Cocaine opens the blood-brain barrier to HIV-1 invasion. F Neurovirol 1998;4: 619-26.

14 So YT, Holtzman DM, Abrams DI, et al. Peripheral neuropathy associated with acquired immunodeficiency
syndrome. Prevalence and clinical features from a pyndrome. Prevalence and clinical features from

15 Childs EA, Lyles RH, Selnes OA, et al. Plasma viral load and CD4 lymphocytes predict HIV-associated dementia and sensory neuropathy. Neurology 1999;52:607-13.

16 Markus R, Brew BJ. HIV-1 peripheral neuropathy and combination antiretroviral therapy. Lancet 1998;352:1906-7.

17 Dannemann B, McCutchan JA, Israelski D, et al, for the California Collaborative Treatment Group. Treatment of toxoplasma encephalitis in patients with AIDS, a randomized trial comparing pyrimethamine plus clindamycin to pyrimethamine plus sulfadiazine. Ann Intern Med 1992. 116:33-43.

18 Girard PM, Landman R, Gaudebout C, et al, for the PRIO Study Group. Dapsone-pyrimethamine compared with aerosolized pentamidine as primary prophylaxis against aerosolized pentamidine as primary prophylaxis against pneumocystis carinii pneumonia and toxoplasm
infection. N Engl $7 \mathrm{Med}$ 1993;328:1514-20.

19 Levy RM, Janssen RS, Bush TJ, et al. Neuroepidemiology of acquired immunodeficiency syndrome. 7 Acquir Immune Defic Syndr Hum Retrovirol 1988;1:31-40.

20 Albrecht H, Hoffmann C, Degen O, et al. Highly active antiretroviral therapy significantly improves the prognosis of patients with HIV-associated progressive multifocal leukoencephalopathy. AIDS 1998;12:1149-54.

21 Clifford DB, Yiannoutsos C, Glicksman M, et al. HAART improves prognosis in HIV-associated progressive multifocal leukoencephalopathy. Neurology 1999;52:623-5. 